\title{
Sind Langschläfer genetisch determiniert?
}

Fragestellung: Gibt es genetische Loci, die mit der Schlafdauer assoziiert sind und stehen diese mit anderen Erkrankungen in Zusammenhang?

Hintergrund: Die nächtliche Schlafdauer gilt als wichtiger Einflussfaktor auf die Leistungsfähigkeit am Tag. Auch liegen viele Hinweise vor, dass eine sehr kurze oder lange Schlafdauer mit verschiedenen Krankheiten assoziiert ist. Während Schlafregulation auf zellulärer Ebene immer besser verstanden ist, liegen zur genetischen Steuerung von Schlaffunktionen und -kenngrößen nur wenige Untersuchungen vor. Zur Schlafdauer gibt es zwar einzelne Berichte, aber keine großen genomweiten Studien.

Patienten und Methodik: In 18 verschiedenen Kollektiven wurde die selbstberichtete Schlafdauer an Wochentagen als Nebenbefund erhoben. Innerhalb

Gottlieb DJ, Hek K, Chen TH et al. Novel loci associated with usual sleep duration: the CHARGE Consortium GenomeWide Association Study. Mol Psychiatry 2015; 200: $1232-9$ der Kohorten wurden Assoziationen zwischen den erhobenen Genotypen und der Schlafdauer ermittelt. Danach wurden alle Datensätze in einer Metaanalyse mit über zwei Millionen Einzel-
Nucleotid Polymorphismen (SNP) getestet und die gefundenen SNP auf ihren Einfluss auf die Genexpression überprüft. Um die SNP-Regionen auf ihre mögliche klinische Funktion einzuschätzen, fand in einem finalen Schritt ein Abgleich mit den veröffentlichten Expressionsdatensets zu Schilddrüsenfunktion, Zuckerhaushalt und psychischen Erkrankungen statt.

Ergebnisse: Zwei voneinander unabhängige Genloci, die mit der berichteten Schlafdauer in Verbindung standen, wurden identifiziert. Eine Region auf Chromosom 2, benachbart zu einem Gen, das als Transkriptionsfaktor (PAX8) follikuläre Schilddrüsenzellen während der Entwicklung stimuliert. In der Analyse einer möglichen Funktion fiel eine Verbindung des Typs, der mit einer langen Schlafdauer korrelierte, mit einer besseren Stoffwechsellage und einem geringen ADHS-Risiko auf. Der zweite Fundort lag auf Chromosom 6 in einer Region zwischen bekannten Genen und korrelierte positiv mit fünf psychischen Erkrankungen.

Schlussfolgerungen: Die Autoren kommen zu dem Schluss, dass auf Grund der Assoziation der Schlafdauer mit verschiedenen relevanten Erkrankungen eine Entschlüsselung gemeinsamer genetischer Korrelate helfen könnte, die Pathophysiologie besser zu verstehen.

\section{- Kommentar von Lukas Frase und Dieter Riemann, Freiburg i. Br. \\ Schlaf - auch genetisch ein komplexes Phänomen}

Schlaf stellt einen komplexen und basalen physiologischen Prozess dar, dessen genaue genetische Regulation nicht vollständig verstanden ist. Einzelne Genloci die zu verschiedenen Charakteristiken von Schlaf beitragen, konnten in den letzten Jahren jedoch identifiziert werden. Die Autoren nutzen vorhandene Datensets aus großangelegten genomweiten Studien, um sich der genetischen Kontrolle der Schlafdauer anzunähern.

Während die geschilderte Methodik der Genomanalyse neuesten technischen Ansprüchen zu genügen scheint, wird die Schlafdauer nur über Selbstbeurteilungsfragebögen erfasst. Eine Objektivierung durch Aktimetrie oder Polysomnografie wird zwar als wünschenswert diskutiert, liegt in der aktuellen Studie jedoch nicht vor. Die Beurteilung des Schlafs bleibt hierdurch annäherungsweise und im Vergleich zur detaillierten Genomanalyse sehr grob.

Zwei verschiedene Genloci werden als signifikant herausgestellt. Da beide Regionen nicht innerhalb eines bekannten Gens liegen, können nur eingeschränkt Rückschlüsse hinsichtlich des zugrunde liegenden Mechanismus gezogen werden. Eine Betrachtung benachbarter Regionen lässt jedoch Spekulationen zu, dass beispielsweise Schilddrüsengewebe eine Rolle in der Etablierung der Schlafdauer spielen könnte. Zusätzlich beschreiben die Autoren eine Assoziation mit verschiedenen psychischen Erkrankungen. Hierbei fällt auf, dass eine vollständige Interpretation des Genoms aktuell nicht zur
Verfügung steht, durch den Vergleich mit hypothesengestützt vorausgewählten Datensets kann auch nur eine Korrelation mit diesen entdeckt werden. Andere mögliche Funktionen der vorgestellten Region werden hierbei möglicherweise übersehen. Zudem könnten auch epigenetische Prozesse eine wichtige Rolle in der Kontrolle der Schlafregulation spielen [1]. Trotz gewisser Einschränkungen stellt die Arbeit einen wichtigen Ausgangspunkt in der Identifizierung der genetischen Grundlagen der Schlafregulation und des Verständnisses der komplexen Zusammenhänge zwischen Schlaf und körperlicher wie psychischer Gesundheit dar.

\section{Referenz: \\ 1. Palagini L et al. Sleep Med Rev 2014; 18: 225 - 35}

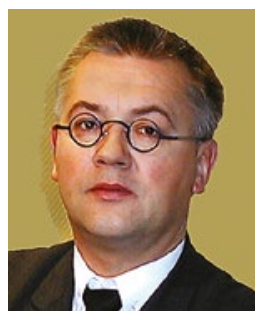

Prof. Dr. Dieter Riemann, Freiburg i. Br.

Universitätsklinik Freiburg

Klinik für Psychiatrie und Psychotherapie

E-Mail: dieter.riemann@uniklinik-freiburg.de 